Interfaces and Free Boundaries 12 (2010), 293-310

DOI $10.4171 / \mathrm{IFB} / 236$

\title{
Nonlinear stability analysis of a two-dimensional diffusive free boundary problem
}

\author{
MICAH WEBSTER \\ Mathematics and Computer Science Department, Goucher College, \\ 1021 Dulaney Valley Road, Baltimore, MD 21286, USA \\ E-mail:micah.webster@goucher.edu \\ PATRICK GUIDOTTI \\ Department of Mathematics, University of California, Irvine, \\ 340 Rowland Hall, Irvine, CA 92697, USA \\ E-mail: gpatrick@math.uci.edu
}

[Received 23 February 2009 and in revised form 25 January 2010]

\begin{abstract}
We explore global existence and stability of planar solutions to a multi-dimensional Case II polymer diffusion model which takes the form of a one-phase free boundary problem with phase onset. Due to a particular boundary condition, convergence cannot be expected on the whole domain. A boundary integral formulation derived in [13] is shown to remain valid in the present context and allows us to circumvent this difficulty by restricting the analysis to the free boundary. The integral operators arising in the boundary integral formulation are analyzed by methods of pseudodifferential calculus. This is possible as explicit symbols are available for the relevant kernels. Spectral analysis of the linearization can then be combined with a known principle of linearized stability [12] to obtain local exponential stability of planar solutions with respect to two-dimensional perturbations.
\end{abstract}

\section{Introduction}

Diffusion in some polymers exhibits anomalous behavior, sometimes called Case II diffusion. Models for its description go back to [1] and [2] and take the form of one phase free boundary problems with kinematic condition. The one-dimensional model of [2] has been analyzed in [3, 4, 5] and has been extended to two dimensions in [10]. These models are characterized by the initial and instantaneous onset of a phase which, mathematically, leads to a singular (initial) boundary value problem for the solvent concentration coupled with an evolution equation of parabolic or HamiltonJacobi type for the front evolution. Their well-posedness has been successfully studied in [6, 8]. The first paper deals with the quasi-stationary approximation while the second treats the full evolutionary problem via an abstract approach developed in [9].

In one space dimension global existence of solutions has been established in the earlier papers mentioned above so that the natural question of their stability with respect to two-dimensional perturbations arises. Formal arguments allowed the authors of [10] to conclude that such solutions are indeed stable with respect to infinitesimal perturbations, albeit in the presence of an initial transient instability. In this paper a rigorous proof is given of the stability of one-dimensional, "flat" for short, solutions for the two-dimensional quasi-stationary approximation. The proof relies on a version of the principle of linearized stability which is valid in the appropriate functional setting.

The framework developed in [6, 8, 9] is not suited for the global well-posedness and stability analysis as it views the free boundary problem as a coupled system for the diffusion of the solvent 
and the motion of the front. The reason lies in the fact that truly two-dimensional solutions, i.e. with nontrivial transversal profiles (see precise formulation in Section 2), even when they converge to a flat solution, only do so on the moving boundary (which itself becomes flat). As this happens, a boundary layer develops in proximity of the fixed boundary where a nonhomogeneous Dirichlet condition is imposed modeling a reservoir kept at constant but nontrivial concentration. This clearly prevents convergence on the whole domain.

Thus we must reformulate the model so as to consider only the dynamics on the free boundary. A natural way to obtain an equivalent system for the boundary effects is to employ a boundary integral formulation. For the equations of interest such a formulation has been derived in [13]. The approach has several advantages for performing nonlinear analysis: Firstly, essentially it turns the problem into a scalar one. Secondly, the concentration on the free boundary can be expressed through an operator depending solely on the free boundary itself. Most importantly, the boundary integral formulation allows us to view the problem as a dynamical system for which we can exploit maximal regularity to obtain global existence results. The key element of many theorems using maximal regularity arguments is the spectral analysis of the generator associated to the linearization. To perform the latter we take the point of view of pseudodifferential operators. This is a viable approach since it allows us to explicitly compute the resolvent and spectrum of the generator needed in order to apply stability theorems.

The nonlinear stability results obtained in this paper rely on linear results that extend the linear ones found in [13]. For the purposes of the latter paper it was sufficient to linearize the integrodifferential equations (18) satisfied by the unknown free boundary in the trivial solution, whereas, in the current paper, understanding of the linearization in any constant (in space) solution is necessary. Thus, while the present paper relies on the boundary integral formulation (BIF) derived in [13], the validity of such BIF still needs to be established in a neighborhood of a more general class of special solutions for it to be useful in the nonlinear stability analysis.

We organize the paper as follows: in Section 2 we formulate the two-dimensional model and briefly discuss the planar or flat solutions. The main result, Theorem 3.1, which asserts that flat solutions are locally exponentially stable with respect to periodic, mean-zero $H^{2}$-perturbations is formulated in Section 3 along with a result about the asymptotic behavior of the free boundary, generalizing known one-dimensional observations. The proof of the main result stretches over the remainder of the paper. Section 4 discusses the boundary integral reformulation, Section 5 deals with its validity close to flat solutions and gives an in-depth analysis of the relevant operators' symbols in order to establish the properties required by the stability theorems of [12]. The nonlinear stability result is then proved in Section 6

\section{Formulation of the 2-D Case II diffusion model}

We assume that a polymer half-space is exposed to a reservoir of solute consisting of small molecules capable of diffusing into it. In the half-space, a sharp interface separates the polymer in two parts. The first is a swollen rubbery region, $\Omega_{t}$, where we assume the solute is free to diffuse, and the second is a glassy region with negligible concentration of solute. We skip the nondimensionalization process and construction of the quasi-stationary approximation and refer to [10] and [6]. The quasi-stationary model for Case II diffusion is given by

$$
\begin{array}{ll}
-\Delta u(t, x, y)=0 & \text { for }(x, y) \in \Omega_{t}, t>0, \\
u(t, x, 0)=g(x)>0 & \text { for } x \in \mathbb{R},
\end{array}
$$




$$
\begin{array}{ll}
-\partial_{\nu_{t}} u(t, x, s(t, x))=\dot{s}(t, x) & \text { for } x \in \mathbb{R}, t>0, \\
\dot{s}(t, x)=\left(\sqrt{1+s_{x}^{2}}+\delta \frac{s_{x x}}{1+s_{x}^{2}}\right) \gamma_{t} u(t, x, s(t, x)) & \text { for } x \in \mathbb{R}, t>0, \\
s(0, x) \equiv 0 & \text { for } x \in \mathbb{R} .
\end{array}
$$

Equation (1) must hold in an unknown, strip-like domain

$$
\Omega_{t}=\left\{(x, y) \in \mathbb{R}^{2}: 0<y<s(t, x)\right\}
$$

with fixed bottom boundary $\Gamma_{0}=\mathbb{R} \times\{0\}$ and moving boundary

$$
\Gamma_{t}=\{(x, s(t, x)): x \in \mathbb{R}\}
$$

for positive time as depicted in Figure 1 We assumed that $\Gamma_{t}$ can be parametrized by an unknown

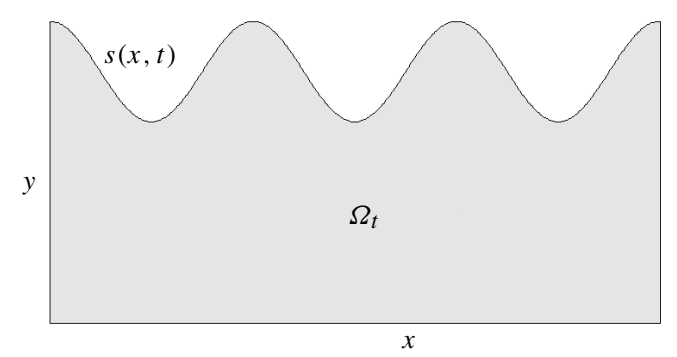

FIG. 1. The setup of the free boundary problem of interest.

smooth function $s$. In addition, $u$ denotes the concentration of solute, $v_{t}$ is the unit outer normal to the free boundary, and $\delta$ is a nonnegative parameter. As hinted by Figure 1 , we will focus on the $x$-periodic case.

A consequence of (1)-(5) combined with the well-posedness for smooth solutions established in [6] is that $\dot{s}(0, x)=g(x)$, and even though this is clearly not a necessary condition, we will at times explicitly add it to the formulation of the equations for the sake of clarity.

When the boundary datum is flat (independent of $x$ ) the system yields a flat-solution pair denoted by $\left(u_{f}(t, y), s_{f}(t)\right)$ or just $\left(u_{f}, s_{f}\right)$. In this case (1)-(5) reduce to the following system:

$$
\begin{array}{ll}
-\partial_{y y} u(t, y)=0, & 0<y<s(t), t>0, \\
u(t, 0)=g_{f}>0, & t>0, \\
\dot{s}(t)=-\partial_{y} u(t, s(t)), & t>0, \\
\dot{s}(t)=u(t, s(t)), & t>0, \\
s(0)=0 . &
\end{array}
$$

Using the Ansatz that $u$ is linear in $y$, it is easy to see that we have a solution pair defined as follows:

$$
u(t, y)=\frac{-g_{f}}{\sqrt{1+2 g_{f} t}} y+g_{f}=: u_{f}(t, y), \quad s(t)=-1+\sqrt{1+2 g_{f} t}=: s_{f}(t) .
$$

The pair $\left(u_{f}, s_{f}\right)$ satisfies $(1)-(5)$, and we should point out that with flat boundary datum we can apply the uniqueness result derived by Guidotti in [6] to justify the simplification made in (6)-(10). 
Observe that, due to condition (2), once we allow $x$-dependence in the boundary datum it is impossible for the solution pair of (1)-(5) to converge to a flat solution pair of $(6)-(10)$ on the whole domain since a boundary layer develops at the bottom boundary. This observation led us to the idea of using a boundary integral formulation (BIF) to analyze the behavior of solution pairs restricted to $\Gamma_{t}$ only.

\section{Main results}

The following main theorem will be proved in Sections 5 and 6 . It relies on the compact reformulation (18) which is indeed shown to be valid in a neighborhood of flat solutions via pseudodifferential calculus analysis in Section 5. It also relies on a version of the principle of linearized stability found in [12] which is briefly described at the beginning of Section 6. The latter section then goes on to prove the validity of the required spectral properties of the linearization by means of pseudodifferential calculus, symbol analysis, and a perturbation argument.

\subsection{Global existence and stability}

The notations for the function spaces used in the formulation of Theorem 3.1 and subsequent Proposition 3.1 are introduced in Subsection 4.1 below.

THEOREM 3.1 (Global existence and stability of flat solutions) Let $g \in H_{p}^{2}([0,1])$ be such that $g>0$. There exist positive constants $\delta_{0}, \omega, \epsilon, K, t^{*}$ and a function $r^{*}$ such that if $\delta \geqslant \delta_{0}$ and $\left\|g-g_{f}\right\|_{H_{p}^{2}}<\epsilon$ then there exists a global solution $s$ to (18) such that

$$
\begin{aligned}
& s-s_{f} \in C^{\alpha}\left(\left[t^{*}, T\right] ; H^{0}\right) \cap C^{1+\alpha}\left(\left[t^{*}, T\right] ; L^{0}\right), \\
& \left\|s(T)-s_{f}(T)\right\|_{H_{p}^{2}} \leqslant K e^{-T \omega}\left\|r^{*}\right\|_{H_{p}^{2}}, \quad \forall T \geqslant t^{*} .
\end{aligned}
$$

Once the asymptotic flattening of the boundary is obtained, it is immediate to recover the convergence of the solute concentration restricted to the free boundary to an $x$-independent one from the boundary integral formulation. We stress again that this convergence does not, however, extend to the whole domain since $u(t, x, 0)=g(x)$.

An important ingredient in the stability result is local existence and continuous dependence on the boundary datum. The result can be found in [13] and is stated here for convenience.

TheOREM 3.2 Let $g \in H_{p}^{2}([0,1])$ be such that $g>0$, and $\delta$ be a positive real number. There exists $T=T\left(s_{0}\right)>0$ and a strict solution

$$
s \in C^{\alpha}\left([0, T] ; H^{+}\right) \cap C^{1+\alpha}\left([0, T] ; L_{2, p}([0,1])\right)
$$

to $[18)$. Furthermore, let $s_{g_{1}}$ and $s_{g_{2}}$ be solutions to $(18)$ with $H_{p}^{2}([0,1])$ boundary data $g_{1}$ and $g_{2}$ respectively. Then there exist $C, r>0$ such that if $\left\|g_{1}-g_{2}\right\|_{H_{p}^{2}([0,1])} \leqslant r$, then

$$
\left\|s_{g_{1}}-s_{g_{1}}\right\|_{C^{\alpha}\left([0, T] ; H_{p}^{2}([0,1])\right)}+\left\|\dot{s}_{g_{1}}-\dot{s}_{g_{2}}\right\|_{C^{\alpha}\left([0, T] ; L_{2, p}([0,1])\right)} \leqslant C\left\|g_{1}-g_{2}\right\|_{H_{p}^{2}([0,1])}
$$

where $H^{+}:=\left\{v \in H_{p}^{2}([0,1]): v \geqslant 0\right\}$. 


\subsection{An asymptotic estimate}

Let $(u(t, x, y), s(t, x))$ be a smooth solution to (1)-5 for $t \in[0, T]$. Then from Green's identity and the parameterization

$$
\Gamma_{t}=\{(x, s(t, x)): x \in[0,1]\}
$$

one computes

$$
0=\int_{\Omega_{t}} y \Delta u \mathrm{~d} z=\int_{0}^{1}[g(x)-(\dot{s}(t, x) s(t, x)+u(x, s(t, x)))] \mathrm{d} x, \quad t \in[0, T],
$$

which yields

$$
\begin{aligned}
\int_{0}^{1} g(x) \mathrm{d} x & =\int_{0}^{1} s(t, x) \dot{s}(x) \mathrm{d} x+\int_{0}^{1} u(x, s(t, x)) \mathrm{d} x, \quad t \in[0, T], \\
\|s\|_{L_{2}}^{2} & =2 t \int_{0}^{1} g(x) \mathrm{d} x-2 \int_{0}^{t} \int_{0}^{1} u(x, s(\tau, x)) \mathrm{d} x \mathrm{~d} \tau, \quad t \in[0, T],
\end{aligned}
$$

and delivers an estimate for the long time behavior of the front.

Proposition 3.1 Let $u \in L_{1}\left([0, \infty) ; C^{2}([0,1) \times[0, \infty), \mathbb{R})\right)$ and $s(\cdot, x) \in C^{1}([0, \infty))$ for all $x \in[0,1)$. Then there exists a function $\eta(t)$ such that

$$
(1-\eta(t)) \sqrt[1 / 2]{2 t \int_{0}^{1} g(x) \mathrm{d} x} \leqslant\|s\|_{L_{2}} \leqslant \sqrt{2 t \int_{0}^{1} g(x) \mathrm{d} x,} \quad t \in[0, T],
$$

where $\lim _{t \rightarrow \infty} \eta(t)=0$.

Proof. The inequality is immediate from (12) with

$$
\eta(t)=\frac{1}{t \int_{0}^{1} g(x) \mathrm{d} x} \int_{0}^{t} \int_{0}^{1} u(x, s(\tau, x)) \mathrm{d} x \mathrm{~d} \tau .
$$

Cohen and Erneux [3] noticed that in the Astarita-Sarti model [2] the free boundary initially grew proportional to $t$, but then eventually switched to a $\sqrt{t}$-behavior. The estimate in the above proposition shows that the two-dimensional Case II diffusion model considered here exhibits the same behavior in the $L_{2}$-norm provided the solution exists for large enough time. Furthermore, Proposition 3.1 demonstrates the asymptotic dependence the free boundary has on the average value of $g$.

\section{Boundary integral formulation}

\section{$4.1 \quad$ Notations and conventions}

Let $\Omega$ be a domain in $\mathbb{R}^{n}$. We denote by $L_{2}(\Omega)$ the usual Hilbert space of square-integrable functions on $\Omega . H^{m}(\Omega)$ will denote the class of all functions defined on $\Omega$ whose first $m$ weak derivatives are in $L_{2}(\Omega)$, and with norm

$$
\|f\|_{H^{m}(\Omega)}^{2}:=\sum_{0 \leqslant|\alpha| \leqslant m}\left\|D^{\alpha} f\right\|_{L_{2}}^{2} .
$$


Denote the Fourier transform on the circle $[0,1]$ by

$$
\left(\mathcal{F}_{x \mapsto k} u\right)(k)=\hat{u}(k)=\int_{0}^{1} e^{-2 \pi i k x} u(x) \mathrm{d} x, \quad k \in \mathbb{Z},
$$

and the Fourier inverse by

$$
\left(\mathcal{F}_{k \mapsto x}^{-1} u\right)(x)=\sum_{k \in \mathbb{Z}} \hat{u}(k) e^{2 \pi i k x}, \quad x \in[0,1] .
$$

Let $H_{p}^{m}=H_{p}^{m}([0,1])$ be the Sobolev spaces on the unit circle, that is,

$$
H_{p}^{m}([0,1])=\left\{u \in L_{2}([0,1]): \sum_{k \in \mathbb{Z}}(1+|k|)^{m}|\hat{u}(k)|^{2}<\infty\right\} .
$$

We denote $H_{p}^{0}$ by $L_{2, p}$ and we set

$$
\|u\|_{H_{p}^{m}}^{2}=\sum_{k \in \mathbb{Z}}(1+|k|)^{m}|\hat{u}(k)|^{2} .
$$

Let $L^{0}$ and $H^{0}$ be the spaces of mean-zero $L_{2, p}$ and $H_{p}^{2}$ functions respectively on [0,1], that is,

$$
L^{0}=\left\{v \in L_{2, p}([0,1]): \hat{v}_{0}=0\right\}, \quad H^{0}=\left\{v \in H_{p}^{2}([0,1]): \hat{v}_{0}=0\right\} .
$$

Due to mathematical conventions, we use $\delta$ as a notation in three different ways, all made clear by the context. The Dirac delta distribution with mass centered at $(x, y)$ is represented by $\delta_{(x, y)}$. The Kronecker delta function is given by $\delta(k)$, and lastly a simple $\delta$ is fixed as a real parameter given in the Case II model.

Let $X$ be a real or complex Banach space with norm $\|\cdot\|$, let $J \subset \mathbb{R}$ be an interval, and define $B(J ; X)$ as the collection of all bounded functions $f: J \rightarrow X$. We endow $B(J ; X)$ with the sup norm

$$
\|f\|_{B(J ; X)}=\sup _{t \in J}\|f(t)\| .
$$

We also define the spaces of bounded continuous, and of $m$ times continuously differentiable functions, as follows:

$$
\begin{gathered}
C_{b}(J ; X)=B(J ; X) \cap C(J ; X), \quad\|f\|_{C_{b}(J ; X)}=\|f\|_{B(J ; X)}, \\
C_{b}^{m}(J ; X)=\left\{f \in C^{m}(J ; X): f^{(k)} \in C_{b}(J ; X), k=0,1, \ldots, m\right\}, \\
\|f\|_{C_{b}^{m}(J ; X)}=\sum_{k=0}^{m}\left\|f^{(k)}\right\|_{B(J ; X) .}
\end{gathered}
$$

The main reason for introducing the previous spaces is to define the spaces of Hölder continuous functions and $C^{\alpha}(J ; X), C^{k+\alpha}(J ; X)$ for $k \in \mathbb{N}, \alpha \in(0,1)$ by

$$
\begin{aligned}
C^{\alpha}(J ; X) & =\left\{f \in C_{b}(J ; X):[f]_{C^{\alpha}(J ; X)}=\sup _{t, s \in J, s<t} \frac{\|f(t)-f(s)\|}{(t-s)^{\alpha}}<\infty\right\}, \\
\|f\|_{C^{\alpha}(J ; X)} & =\|f\|_{B(J ; X)}+[f]_{C^{\alpha}}, \\
C^{k+\alpha}(J ; X) & =\left\{f \in C_{b}^{k}(J ; X): f^{(k)} \in C^{\alpha}(J ; X)\right\}, \\
\|f\|_{C^{k+\alpha}(J ; X)} & =\|f\|_{C_{b}^{k}(J ; X)}+\left[f^{(k)}\right]_{C^{\alpha}(J ; X)} .
\end{aligned}
$$




\subsection{Reformulation}

Boundary integral formulations of (free) boundary value problems rely on classical integral representations of solutions by means of layer potentials derived from explicit knowledge of fundamental solutions and Green's formula. In the two-dimensional $x$-periodic case considered here a fundamental solution for

$$
-\Delta G=\delta_{(x, y)}, \quad(x, y) \in[0,1) \times[0, \infty),
$$

can be explicitly computed:

$$
G(x, y)=\frac{1}{2 \pi} \log \left|1+e^{-4 \pi y}-2 \cos (2 \pi x) e^{-2 \pi y}\right|
$$

as pointed out in [7]. We omit the derivation of the BIF and refer to [13] instead.

Let $s \in H_{p}^{2}([0,1]), \dot{s} \in L_{2, p}([0,1]), g \in H_{p}^{2}([0,1])$ and consider the boundary integral equation for $\Psi[s](x):=u(x, s(x))$ given by

$$
\left(\mathrm{id}+\mathcal{I}_{s}\right)[\Psi]=\mathcal{R}_{3}(g, s, \dot{s})
$$

where

$$
\begin{aligned}
\mathcal{I}_{S}[\Psi](x)= & \int_{0}^{1}\left(-s_{x}(\tilde{x}), 1\right) \nabla G(x-\tilde{x}, s(x)-s(\tilde{x})) \Psi(\tilde{x}) \mathrm{d} \tilde{x} \\
& -\int_{0}^{1} G(x-\tilde{x}, s(x)) \mathcal{N}\left[\int_{0}^{1}\left(-s_{\tilde{x}}(z), 1\right) \cdot \nabla G(\tilde{x}-z,-s(z)) \Psi(z) \mathrm{d} z\right] \mathrm{d} \tilde{x}
\end{aligned}
$$

and

$$
\begin{aligned}
\mathcal{N}[h]= & \mathcal{F}^{-1}[2 \pi|k|] \mathcal{F}[h], \\
\mathcal{R}_{1}(g, s, \dot{s})(x)= & \int_{0}^{1}[g(\tilde{x})+(G(x-\tilde{x},-s(\tilde{x}))-G(x-\tilde{x}, 0)) \dot{s}(\tilde{x})] \mathrm{d} \tilde{x}, \\
\mathcal{R}_{2}(g, s, \dot{s})(x)= & \int_{0}^{1}[G(x-\tilde{x}, s(x))-G(x-\tilde{x}, s(x)-s(\tilde{x}))] \dot{s}(\tilde{x}) \mathrm{d} \tilde{x}, \\
& +\int_{0}^{1} g(\tilde{x}) \frac{\partial G}{\partial y}(x-\tilde{x}, s(x)) \mathrm{d} \tilde{x}, \\
\mathcal{R}_{3}(g, s, \dot{s})= & \int_{0}^{1} G(\cdot-\tilde{x}, s(\cdot)) \mathcal{N}\left[\mathcal{R}_{1}(g, s, \dot{s})(\cdot)\right](\tilde{x}) \mathrm{d} \tilde{x}+\mathcal{R}_{2}(g, s, \dot{s}) .
\end{aligned}
$$

Then the equivalent boundary reformulation of (1)-(5) is given by

$$
\begin{cases}\dot{s}(t, x)=\left(\sqrt{1+s_{x}^{2}}+\delta \frac{s_{x x}}{1+s_{x}^{2}}\right) \Psi[s], & (t, x) \in(0, \infty) \times[0,1], \\ s(0, x)=0, & x \in[0,1], \\ \Psi[s(0, \cdot)](x)=g, & x \in[0,1],\end{cases}
$$

where $\Psi[s]=\left(\mathrm{id}+\mathcal{I}_{s}\right)^{-1} \mathcal{R}_{3}(g, s, \dot{s})$. The crucial question, on which hinges the validity of the reformulation, is whether or not the integral operator

$$
I_{s}=\mathrm{id}+\mathcal{I}_{s}
$$


possesses an inverse. In [13] it has been shown that this is the case for small time or, equivalently, in a neighborhood of $s \equiv 0$. In this paper we show that $(18)$ is also well-defined when $s$ is close to being flat so that the nonlinear stability theorem given in [12] can be applied to it.

\section{Validity of the boundary integral formulation}

In this section, we provide the proper context and relevant definitions to view the operators appearing in the BIF as pseudodifferential operators with known symbols. This makes it possible to show that reformulation (18) is well-defined. Knowledge of the symbols will also be essential later in proving Theorem 3.1 .

\subsection{Pseudodifferential operators with nonregular symbols}

For a function $\sigma:[0,1] \times[0,1] \times \mathbb{Z} \rightarrow \mathbb{C}$ define

$$
\begin{aligned}
& \langle k\rangle=\left(1+|k|^{2}\right)^{1 / 2}, \\
& \triangle_{k} \sigma(x, y, k)=\sigma(x, y, k+1)-\sigma(x, y, k), \\
& \triangle_{k}^{\alpha} \sigma(x, y, k)=\sum_{\beta \leqslant \alpha}(-1)^{|\alpha-\beta|}\left(\begin{array}{l}
\alpha \\
\beta
\end{array}\right) \sigma(x, y, k+\beta) .
\end{aligned}
$$

Definition 5.1 For $\tau \geqslant 0, S_{\rho, \delta ; \tau}^{m}$ is defined as the set of symbols $\sigma(x, y, k)$ which have continuous derivatives satisfying

$$
\left|\triangle_{k}^{\alpha} \partial_{x}^{\beta} \partial_{y}^{\beta^{\prime}} \sigma\right| \leqslant C\langle k\rangle^{m+\delta\left|\beta+\beta^{\prime}\right|-\rho|\alpha|}
$$

for any $\alpha$ and $\left|\beta+\beta^{\prime}\right| \leqslant[\tau]$ and

$$
\left|\triangle_{k}^{\alpha} \partial_{x}^{\beta} \partial_{y}^{\beta^{\prime}} \sigma(x, y, k)-\triangle_{k}^{\alpha} \partial_{x}^{\beta} \partial_{y}^{\beta^{\prime}} \sigma\left(x^{\prime}, y^{\prime}, k\right)\right| \leqslant C\left(\left|x-x^{\prime}\right|^{\dot{\tau}}+\left|y-y^{\prime}\right|^{\dot{\tau}}\right)\langle k\rangle^{m+\delta \tau-\rho \alpha}
$$

for $\left|\beta+\beta^{\prime}\right|=[\tau], \tau>0$ and $\left|x-x^{\prime}\right| \leqslant 1,\left|y-y^{\prime}\right| \leqslant 1$ where $C$ is a constant depending on $\alpha, \beta$ and $\beta^{\prime}$, and $\dot{\tau}=\tau-[\tau]$.

For $\sigma(x, y, k) \in S_{1, \delta^{\prime} ; \tau}^{0}$ we define

$$
O p(\sigma)[f](x)=\int_{0}^{1} \sum_{k \in \mathbb{Z}} \sigma(x, y, k) e^{2 \pi i k(x-y)} f(y) \mathrm{d} y .
$$

The operator $O p(\sigma)$ is a bounded operator from $L_{q}$ to $L_{q}$, provided $0 \leqslant \delta^{\prime}<1$ and $0<\tau \leqslant 1$. Since we restrict ourselves to the $L_{2, p}([0,1])$ context, the conditions (19)-20] in Definition 5.1 need only hold for $|\alpha|=0,1,2$ (see [11]). Recall our logarithmic kernel, $G$, in the boundary integral formulation is defined by

$$
G(x, y)=\frac{1}{2 \pi} \log \left|1+e^{-4 \pi y}-2 \cos (2 \pi x) e^{-2 \pi y}\right| .
$$

To perform the stability analysis we need to understand the symbol when $y$ varies. In the following definition we summarize the results proved in [7] 
Definition 5.2 (Theorem 8 in [7]) Let $s \in H_{p}^{2}([0,1])$.

(a) We have

$$
\int_{0}^{1} G_{y}(x-\tilde{x}, s(x)-s(\tilde{x})) f(\tilde{x}) \mathrm{d} \tilde{x}=O p\left(a_{K}\right)[f](x)
$$

for any $f \in H_{p}^{1}([0,1])$, where $a_{K}=a_{K}(k, x, \tilde{x})$ is given by

$$
a_{K}(k, x, \tilde{x})= \begin{cases}\exp (-2 \pi|k|(s(x)-s(\tilde{x}))), & k \in \mathbb{Z}, s(x)-s(\tilde{x}) \geqslant 0, \\ -2 \delta(k)-\exp (2 \pi|k|(s(x)-s(\tilde{x}))), & k \in \mathbb{Z}, s(x)-s(\tilde{x})<0 .\end{cases}
$$

(b) We have

$$
\int_{0}^{1} G(x-\tilde{x}, s(x)-s(\tilde{x})) f(\tilde{x}) \mathrm{d} \tilde{x}=O p\left(a_{G}\right)[f](x)
$$

for any $f \in L_{2, p}([0,1])$, where $a_{G}=a_{G}(k, x, \tilde{x})$ is given by

$$
a_{G}(k, x, \tilde{x})= \begin{cases}\frac{\exp (-2 \pi|k|[s(x)-s(\tilde{x})])}{2 \pi|k|}, & s(x)-s(\tilde{x}) \geqslant 0, \\ \frac{\exp (2 \pi|k|[s(x)-s(\tilde{x})])}{2 \pi|k|}+e^{2 \pi i k \tilde{x}} \hat{c}(k, \tilde{x}), & s(x)-s(\tilde{x})<0,\end{cases}
$$

for $k \in \mathbb{Z}^{*}$ and where the correction term $\hat{c}$ is given by

$$
\hat{c}(\cdot, \tilde{x})=\mathcal{F}_{x \mapsto k}\left[-2(s(\cdot)-s(\tilde{x})) \chi_{[s(\cdot)-s(\tilde{x})<0]} .\right.
$$

(c) We have

$$
\int_{0}^{1} G_{x}(x-\tilde{x}, s(x)-s(\tilde{x})) f(\tilde{x}) \mathrm{d} \tilde{x}=O p\left(a_{H}\right)[f](x)
$$

for any $f \in H_{p}^{1}([0,1])$, where $a_{H}=a_{H}(k, x, \tilde{x})$ is given by

$$
a_{H}(k, x, \tilde{x})= \begin{cases}-i \operatorname{sgn}(k) \exp (-2 \pi|k|[s(x)-s(\tilde{x})]), & k \in \mathbb{Z}, s(x)-s(\tilde{x}) \geqslant 0, \\ -i \operatorname{sgn}(k) \exp (2 \pi|k|[s(x)-s(\tilde{x})]), & k \in \mathbb{Z}, s(x)-s(\tilde{x})<0 .\end{cases}
$$

Next we define the core operators associated with the Fréchet derivative of the generator used in the stability result.

Definition 5.3 Let $f, s \in H_{p}^{2}([0,1])$. We define

$$
\operatorname{DOp}_{(s(\cdot)-s(\tilde{x}))}^{f}\left(a_{G}^{+,-}\right): H_{p}^{2}([0,1]) \rightarrow L_{2, p}([0,1])
$$

by setting

$$
\begin{array}{r}
\operatorname{DOp}_{(s(\cdot)-s(\tilde{x}))}^{f}\left(a_{G}^{+}\right)[r]=\int_{0}^{1} \sum_{k \in \mathbb{Z}^{*}}-e^{-2 \pi|k|[s(\cdot)-s(\tilde{x})]} e^{2 \pi i k(\cdot-\tilde{x})}[r(\cdot)-r(\tilde{x})] f(\tilde{x}) \mathrm{d} \tilde{x} \\
\\
\text { if } s(\cdot)-s(\tilde{x}) \geqslant 0,
\end{array}
$$




$$
\begin{aligned}
D O p_{(s(\cdot)-s(\tilde{x}))}^{f}\left(a_{G}^{-}\right)[r]= & \int_{0}^{1} \sum_{k \in \mathbb{Z}^{*}} e^{2 \pi|k|[s(\cdot)-s(\tilde{x})]} e^{2 \pi i k(\cdot-\tilde{x})}[r(\cdot)-r(\tilde{x})] f(\tilde{x}) \mathrm{d} \tilde{x}, \\
& +\int_{0}^{1} \sum_{k \in \mathbb{Z}^{*}} e^{2 \pi i k \tilde{x}} \mathcal{F}_{\cdot \mapsto k}\left[-2(r(\cdot)-r(\tilde{x})) \chi_{[s(\cdot)-s(\tilde{x})<0]}\right] f(\tilde{x}) \mathrm{d} \tilde{x} \\
& \text { if } s(\cdot)-s(\tilde{x})<0 .
\end{aligned}
$$

Moreover, we define

$$
\operatorname{DOp}_{(s(\cdot)-s(\tilde{x}))}^{f}\left(a_{K}\right): H_{p}^{2}([0,1]) \rightarrow L_{2, p}([0,1])
$$

by

$$
\operatorname{DOp}_{(s(\cdot)-s(\tilde{x}))}^{f}\left(a_{K}\right)[r]=\int_{0}^{1} \sum_{k \in \mathbb{Z}^{*}}-2 \pi|k| e^{-2 \pi|k||s(\cdot)-s(\tilde{x})|} e^{2 \pi i k(\cdot-\tilde{x})}[r(\cdot)-r(\tilde{x})] f(\tilde{x}) \mathrm{d} \tilde{x} .
$$

Finally, we define

$$
D O p_{(s(\cdot)-s(\tilde{x}))}^{f}\left(a_{H}\right): H_{p}^{2}([0,1]) \rightarrow L_{2, p}([0,1])
$$

by

$$
\operatorname{DOp}_{(s(\cdot)-s(\tilde{x}))}^{f}\left(a_{H}\right)[r]=\int_{0}^{1} \sum_{k \in \mathbb{Z}^{*}} 2 \pi i k e^{-2 \pi|k||s(\cdot)-s(\tilde{x})|} e^{2 \pi i k(\cdot-\tilde{x})}[r(\cdot)-r(\tilde{x})] f(\tilde{x}) \mathrm{d} \tilde{x} .
$$

Using Definitions 5.15 we can view the operators and their corresponding Fréchet derivatives in the BIF as nonregular pseudodifferential operators. Furthermore, the linearization of the BIF about $s=s_{0}$ satisfies the necessary mapping and Lipschitz continuity properties to apply maximal regularity existence theorems (see [13]). We continue by focusing on the situation when $s$ is nearly flat.

\subsection{Linearization in flat solutions}

Here we compute the Fréchet derivative of the BIF for $\gamma_{t} u$ evaluated at $s=s_{f}$. We begin our investigation by introducing the notation

$$
O p_{f(x, \tilde{x})}\left(a_{G}\right), O p_{f(x, \tilde{x})}\left(a_{K}\right), O p_{f(x, \tilde{x})}\left(a_{H}\right)
$$

for the symbols of the integral operators with kernels

$$
G(x-\tilde{x}, f(x, \tilde{x})), G_{y}(x-\tilde{x}, f(x, \tilde{x})), G_{x}(x-\tilde{x}, f(x, \tilde{x})),
$$

respectively. Let $\epsilon, T>0, s, r \in C^{\alpha}\left([0, T] ; H_{p}^{2}\right) \cap C^{1+\alpha}\left([0, T] ; L_{2, p}\right), g \in H_{p}^{2}([0,1])$ and

$$
\begin{aligned}
B[s](\tilde{x})= & \mathcal{N}\left[\int_{0}^{1}\left(-s_{z}(z), 1\right) \cdot \nabla G(\tilde{x}-z,-s(z)) \Psi[s](z) \mathrm{d} z\right], \\
I_{s}[\Psi](x)= & \Psi[s](x)+\int_{0}^{1}\left(-s_{x}(\tilde{x}), 1\right) \nabla G(x-\tilde{x}, s(x)-s(\tilde{x})) \Psi[s](\tilde{x}) \mathrm{d} \tilde{x} \\
& -\int_{0}^{1} G(x-\tilde{x}, s(x)) B[s](\tilde{x}) \mathrm{d} \tilde{x},
\end{aligned}
$$


and

$$
D \Psi .=\lim _{\epsilon \rightarrow 0} \frac{\Psi[\cdot+\epsilon r]-\Psi[\cdot]}{\epsilon} .
$$

Then $D \Psi_{s}$ must satisfy the following equation (see [13] for the details):

$$
I_{s} D \Psi_{s}=\left(D \mathcal{R}^{1}-D I_{s}\right)[r]+D \mathcal{R}^{2}[\dot{r}]
$$

where

$$
\begin{aligned}
D I_{s}[r](x)= & D O p_{(s(x)-s(\tilde{x}))}^{\Psi[s]}\left(a_{K}\right)[r](x)+D O p_{(s(x)-s(\tilde{x}))}^{-s_{x} \Psi[s]}\left(a_{H}\right)[r](x) \\
& -D O p_{s(x)}^{B[s]}\left(a_{G}\right)[r](x)+O p_{s(x)-s(\tilde{x})}\left(a_{H}\right)\left[-r_{x} \Psi[s]\right](x) \\
& -O p_{s(x)}\left(a_{G}\right)\left[\mathcal { N } \left[D O p_{-s(z)}^{\Psi[s]}\left(a_{K}\right)[r](\tilde{x})+D O p_{-s(z)}^{-s_{x} \Psi[s]}\left(a_{H}\right)[r](\tilde{x})\right.\right. \\
& \left.\left.+O p_{-s(z)}\left(a_{H}\right)\left[-r_{z} \Psi[s]\right](\tilde{x})\right]\right](x) \\
D \mathcal{R}^{1}[r](x)= & D O p_{s(x)}^{g}\left(a_{K}\right)[r](x)+D O p_{s(x)}^{\mathcal{N}\left(\mathcal{R}_{1}(g, s, \dot{s})\right)}\left(a_{G}\right)[r](x) \\
& +O p_{s(x)}\left(a_{G}\right)\left[\mathcal{N}\left[D O p_{-s(z)}^{\dot{s}(z)}\left(a_{G}\right)[r](\tilde{x})\right](x)\right. \\
& +\left(D O p_{s(x)}^{\dot{s}}\left(a_{G}\right)-D O p_{s(x)-s(\tilde{x})}^{\dot{s}}\left(a_{G}\right)\right)[r](x) \\
D \mathcal{R}^{2}[\dot{r}](x)= & \left(O p_{s(x)}\left(a_{G}\right)-O p_{s(x)-s(\tilde{x})}\left(a_{G}\right)\right)[\dot{r}](x) \\
& +O p_{s(x)}\left(a_{G}\right)\left[\mathcal{N}\left[\left(O p_{-s(z)}\left(a_{G}\right)-O p_{0}\left(a_{G}\right)\right)[\dot{r}](\tilde{x})\right]\right](x)
\end{aligned}
$$

which gives

$$
D \Psi_{s}[r, \dot{r}]=I_{s}^{-1}\left[D \mathcal{R}^{1}-D I_{s}\right][r]+I_{s}^{-1}\left[D \mathcal{R}^{2}\right][\dot{r}]=: D \Psi_{s}^{1}[r]+D \Psi_{s}^{2}[\dot{r}]
$$

as soon as $I_{s}^{-1}$ exists. (22) is an explicit representation of the Fréchet derivative of $\Psi[s]$ and is most useful in computing the spectrum of the linearization of (18) about $s=s_{f}$.

\subsection{Proof of validity for BIF}

We are now in a position to prove that the BIF and its Fréchet derivative are well-defined in an $H_{p}^{2}$-neighborhood of $s=s_{f}$. We state and prove the theorem based on a small technical lemma that we postpone until after the proof of the theorem.

THEOREM 5.1 Let $T>0$ and define

$$
Y^{0}=\left\{f: f(t, \cdot) \in H^{0}, \dot{f}(t, \cdot) \in L^{0}, \forall t \in[0, T]\right\} .
$$

If $g \equiv g_{f}>0, s=s_{f}$ and $\dot{s}=\dot{s_{f}}$ then

$$
\begin{aligned}
D \Psi_{s}\left(s_{f}\right): Y^{0} \rightarrow & L_{2, p}, \\
D \Psi_{s}\left(s_{f}\right)[r]= & \dot{s_{f}} \mathcal{F}^{-1}\left(\frac{e^{-4 \pi|k| s_{f}}-e^{-2 \pi|k| s_{f}}+2 \pi|k| e^{-2 \pi|k| s_{f}}-4 \pi|k|}{2+e^{-4 \pi|k|}}\right) \mathcal{F}(r) \\
& +\mathcal{F}^{-1}\left(\left[\frac{e^{-2 \pi|k| s_{f}}-1}{2+e^{-4 \pi|k| s_{f}}}\right]\left[\frac{1+2 \pi|k| e^{-2 \pi|k| s_{f}}}{2 \pi|k|}\right]\right) \mathcal{F}(\dot{r})
\end{aligned}
$$

is a linear operator and there exists an $\eta>0$ such that, for all $s \in \mathbb{B}_{H^{0}}\left(s_{f}, \eta\right), D \Psi_{s}$ is given by [22]. 
Proof. Let $g=g_{f}, s=s_{f}, \dot{s}=\dot{s_{f}}$ and $r \in Y^{0}$. Observe

$$
\mathcal{F}\left[\int_{0}^{1} G\left(\xi-\tilde{\xi}, s_{f}\right) \mathcal{N}[f] \mathrm{d} \tilde{\xi}\right]=e^{-2 \pi|k| s_{f}} \mathcal{F}[f](k)
$$

and by Lemma 5.1] $\Psi\left[s_{f}\right]$ is flat, or in other words constant in the spatial variable. A straightforward calculation gives

$$
\begin{aligned}
\left.D O p_{s(x)-s(\tilde{x})}^{\Psi[s]}\left(a_{K}\right)[r](x)\right|_{s=s_{f}} & =\int_{0}^{1} \sum_{k \in \mathbb{Z}^{*}}(-2 \pi|k|) e^{2 \pi i k(x-\tilde{x})}(r(x)-r(\tilde{x})) \Psi\left[s_{f}\right] \mathrm{d} \tilde{x} \\
& =\Psi\left[s_{f}\right] \mathcal{N}[r](x), \\
\left.O p_{s(x)-s(\tilde{x})}\left(a_{H}\right)\left[-r_{x} \Psi[s]\right](x)\right|_{s=s_{f}} & =\Psi\left[s_{f}\right]\left(\mathcal{F}^{-1}(-2 \pi|k|) \mathcal{F}(r)\right)(x), \\
\left.D O p_{-s(z)}^{\Psi[s]}\left(a_{K}\right)[r](\tilde{x})\right|_{s=s_{f}} & =\Psi\left[s_{f}\right]\left(\mathcal{F}^{-1}(-2 \pi|k|) e^{-2 \pi|k| s_{f}} \mathcal{F}(r)\right)(\tilde{x}), \\
\left.D O p_{s(x)}^{B[s]}\left(a_{G}\right)[r](x)\right|_{s=s_{f}} & =0, \\
\left.O p_{-s(z)}\left(a_{H}\right)\left[-r_{z} \Psi[s]\right](\tilde{x})\right|_{s=s_{f}} & =\Psi\left[s_{f}\right]\left(\mathcal{F}^{-1}(-2 \pi|k|) e^{-2 \pi|k| s_{f}} \mathcal{F}(r)\right)(\tilde{x}) .
\end{aligned}
$$

Using $\left[23\right.$ along with the definition of $D I_{s}[r]$ produces

$$
\left.D I_{s}[r]\right|_{s=s_{f}}=\Psi\left[s_{f}\right] \mathcal{F}^{-1}\left(4 \pi|k|-2 \pi|k| e^{-2 \pi|k| s_{f}}\right) \mathcal{F}(r) .
$$

To calculate $D \mathcal{R}^{1}[r]$, first notice $\mathcal{R}_{1}\left(g_{f}, s_{f}, \dot{s_{f}}\right)$ is constant so that

$$
\mathcal{N}\left[\left.\mathcal{R}_{1}(g, s, \dot{s})\right|_{\left.\left(g=g_{f}, s=s_{f}, \dot{s}=s_{f}\right)\right)}\right] \equiv 0 .
$$

Computing the rest of the terms in the definition of $D \mathcal{R}^{1}[r]$ and $D \mathcal{R}^{2}[\dot{r}]$ yields

$$
\begin{aligned}
& D \mathcal{R}^{1}[r]=\dot{s_{f}} \mathcal{F}^{-1}\left(e^{-4 \pi|k| s_{f}}-e^{-2 \pi|k| s_{f}}\right) \mathcal{F}(r), \\
& D \mathcal{R}^{2}[\dot{r}]=\mathcal{F}^{-1}\left(\frac{e^{-2 \pi|k| s_{f}}-1}{2 \pi|k|}+e^{-4 \pi|k| s_{f}}-e^{-2 \pi|k| s_{f}}\right) \mathcal{F}(\dot{r})
\end{aligned}
$$

Combining 21], 24], 25] and 26) one computes

$$
\begin{aligned}
I_{s_{f}} D \Psi_{s_{f}}[r, \dot{r}]= & \mathcal{F}^{-1}\left(\dot{s_{f}}\left(e^{-4 \pi|k| s_{f}}-e^{-2 \pi|k| s_{f}}\right)+\Psi\left[s_{f}\right]\left(2 \pi|k| e^{-2 \pi|k| s_{f}}-4 \pi|k|\right)\right) \mathcal{F}(r) \\
& +\mathcal{F}^{-1}\left(\frac{e^{-2 \pi|k| s_{f}}-1}{2 \pi|k|}+e^{-4 \pi|k| s_{f}}-e^{-2 \pi|k| s_{f}}\right) \mathcal{F}(\dot{r})
\end{aligned}
$$

where

$$
I_{s_{f}} D \Psi_{s_{f}}[r, \dot{r}]=\left.I_{s} D \Psi_{s}[r, \dot{r}]\right|_{\left(g=g_{f}, s=s_{f}, \dot{s}=s_{f}\right)} .
$$

In Lemma 5.1 we demonstrate that $I_{s}$ is invertible at $s=s_{f}$ and the inverse operator is given by

$$
v \mapsto \mathcal{F}^{-1}\left[(1-\delta(k)) \frac{1}{2+e^{-4 \pi|k| s_{f}}}+\delta(k)\right] \mathcal{F}[v] .
$$

Furthermore, $\Psi\left[s_{f}\right]$ is a smooth solution to the boundary integral formulation and $\left(s_{f}, \Psi\left[s_{f}\right]\right)$ satisfies (6)-(10). Thus by the uniqueness result in [6],

$$
\Psi\left[s_{f}\right]=u_{f}=\dot{s_{f}} .
$$


Putting everything together gives

$$
\begin{aligned}
\left.D \Psi_{s}[r, \dot{r}]\right|_{s=s_{f}}= & \dot{s_{f}} \mathcal{F}^{-1}\left(\frac{e^{-4 \pi|k| s_{f}}-e^{-2 \pi|k| s_{f}}+2 \pi|k| e^{-2 \pi|k| s_{f}}-4 \pi|k|}{2+e^{-4 \pi|k|}}\right) \mathcal{F}(r) \\
& +\mathcal{F}^{-1}\left(\left[\frac{e^{-2 \pi|k| s_{f}}-1}{2+e^{-4 \pi|k| s_{f}}}\right]\left[\frac{1+2 \pi|k| e^{-2 \pi|k| s_{f}}}{2 \pi|k|}\right]\right) \mathcal{F}(\dot{r}) .
\end{aligned}
$$

Using Theorems 5.2 and 5.3 in [13] and Lemma 5.1 there exists $\eta>0$ such that $I_{s}^{-1}$ exists provided $s \in \mathbb{B}_{H^{0}}\left(s_{f}, \eta\right)$, so that $D \Psi_{s}$ is given by [22) in an $\eta$-neighborhood of $s_{f}$.

Lemma 5.1 Let $s=s_{f}, \dot{s}=\dot{s_{f}}$, and $g=g_{f}$. Then $\Psi\left[s_{f}\right]$ is the smooth, flat solution to the equation

$$
I_{s_{f}}[\Psi]=\mathcal{R}_{3}\left(g_{f}, s_{f}, \dot{s_{f}}\right)
$$

where

$$
I_{s_{f}}: H_{p}^{m} \rightarrow H_{p}^{m}, \quad v \mapsto\left(2 \mathrm{id}+\mathcal{F}^{-1}\left[(1-\delta(k)) e^{-4 \pi|k| s_{f}}-\delta(k)\right] \mathcal{F}\right)[v] .
$$

Moreover, $I_{s_{f}}$ is an invertible operator and its inverse is given by

$$
v \mapsto \mathcal{F}^{-1}\left[(1-\delta(k)) \frac{1}{2+e^{-4 \pi|k| s_{f}}}+\delta(k)\right] \mathcal{F}[v] .
$$

Proof. Let $s=s_{f}, \dot{s}=\dot{s_{f}}$, and $g=g_{f}$ and evaluate (id $\left.+\mathcal{I}_{s}\right)[\Psi]$. A straightforward calculation using Definition 5.2 and the Fourier transform completes the proof.

\section{Proof of global existence}

In this final section we begin by briefly discussing asymptotic stability and the principle of linearized stability. We then prove Theorem 3.1 through a series of technical lemmas which verify the assumptions of Theorem 9.1.2 in [12].

\subsection{A principle of linearized stability}

Consider the problem

$$
\begin{aligned}
\dot{u}(t) & =F(u(t)), \quad t \geqslant 0, \\
u(0) & =u_{0},
\end{aligned}
$$

where $F: D \rightarrow X$, and suppose $\bar{u}$ is a stationary solution. The stationary solution is said to be stable if, for each $\epsilon>0$, there is a $\delta>0$ such that, for $\left\|u_{0}-\bar{u}\right\|_{D} \leqslant \delta$, the interval of existence for the solution, $u$, is $[0, \infty)$ and $\left\|u\left(t ; u_{0}\right)-\bar{u}\right\|_{D} \leqslant \epsilon$ for all $t>0$. In addition, the stationary solution is said to be asymptotically stable if it is stable and

$$
\lim _{t \rightarrow \infty}\left\|u\left(t ; u_{0}\right)-\bar{u}\right\|_{D}=0
$$

uniformly for $u_{0}$ in a neighborhood of $\bar{u}$. Under the key assumptions that $A=F^{\prime}(0)$ be locally Lipschitz in a neighborhood of the null solution, that it be a sectorial operator from $D(A)$ to $X$, and that

$$
\sup \left\{\operatorname{Re}(\lambda): \lambda \in \sigma\left(F^{\prime}(0)\right)\right\}=-\omega_{0}<0,
$$


Lunardi proved that the trivial solution to 28$)$ is exponentially asymptotically stable in $D$. That is, for every $\omega \in\left[0, \omega_{0}\right)$ there are $r, M>0$ such that if $\left\|u_{0}\right\|_{D} \leqslant r$, then the interval of existence is $[0, \infty)$ and

$$
\left\|u\left(t ; u_{0}\right)\right\|_{D} \leqslant M e^{-\omega t}\left\|u_{0}\right\|_{D}, \quad t \geqslant 0
$$

The most challenging assumption of the principle of linearized stability is the spectral condition. It will become evident that defining the operators in terms of the Fourier transform is essential.

LEMMA 6.1 The operator

$$
B: L^{0} \rightarrow L^{0}, \quad v \mapsto\left(\mathrm{id}-D \Psi_{s_{f}}^{(2)}\right)[v],
$$

is invertible and the inverse is given by

$$
B^{-1}[w]=\mathcal{F}^{-1}\left(\frac{2 \pi|k|\left(2+e^{-2 \pi|k| s_{f}}\right)}{1+4 \pi|k|+(2 \pi|k|-1) e^{-2 \pi|k| s_{f}}}\right) \mathcal{F}[w]
$$

for every $w \in L^{0}$.

Proof. Let $v, w \in L^{0}$ and consider

$$
B[v]=w .
$$

From Theorem 5.1 taking the Fourier transform of both sides yields

$$
\left(1-\left[\frac{e^{-2 \pi|k| s_{f}}-1}{2+e^{-4 \pi|k| s_{f}}}\right]\left[\frac{1+2 \pi|k| e^{-2 \pi|k| s_{f}}}{2 \pi|k|}\right]\right) \mathcal{F}[v]=\mathcal{F}[w] .
$$

Then solving for $\mathcal{F}[v]$ yields

$$
\mathcal{F}[v]=\frac{2 \pi|k|\left(2+e^{-2 \pi|k| s_{f}}\right)}{1+4 \pi|k|+(2 \pi|k|-1) e^{-2 \pi|k| s_{f}}} \mathcal{F}[w]
$$

and inversion of the Fourier transform yields the claim.

LeMMa 6.2 Consider

$$
\begin{cases}\dot{s}(t, x)=F[s](t, x), & (t, x) \in(0, \infty) \times[0,1], \\ s(0, x)=0, & x \in[0,1] \\ \Psi[s(0, \cdot)](x)=g(x), & x \in[0,1]\end{cases}
$$

where

$$
F[s]=\left(\sqrt{1+s_{x}^{2}}+\delta \frac{s_{x x}}{1+s_{x}^{2}}\right) \Psi[s]
$$

and $g \in H_{p}^{2}([0,1])$ is such that $g>0$. Let $\left(u_{f}, s_{f}\right)$ be the flat solution pair corresponding to the boundary datum $g_{f}:=\int g$ and define the linear operator, $\mathcal{G}$, from $H^{0}$ to $L^{0}$ as follows:

$$
\mathcal{G}[r]=\left(\mathrm{id}-D \Psi_{s_{f}}^{(2)}\right)^{-1}\left[\delta u_{f} \Delta[r]+D \Psi_{s}\left(s_{f}\right)^{(1)}[r]\right] .
$$


Then the linearization about $s=s_{f}$ is given by

$$
\begin{cases}\dot{r}=\mathcal{G}[r] & \text { in }(0, \infty) \times[0,1], \\ r(0, x)=0 & \text { for } x \in[0,1], \\ \Psi[r(0)](x)=g(x)-g_{f} & \text { for } x \in[0,1] .\end{cases}
$$

Furthermore, $\mathcal{G}$ is a sectorial operator from $H^{0}$ to $L^{0}$ and there exist $\omega_{0}, \delta_{0}>0$ such that for $\delta>\delta_{0}$ we have $\sup \{\operatorname{Re}(\sigma(\mathcal{G}))\} \leqslant-\omega_{0}<0$.

Proof. Let $T>0, s-s_{f} \in C^{1+\alpha}\left([0, T] ; L^{0}\right) \cap C^{\alpha}\left([0, T] ; H^{0}\right)$, and

$$
r=s-s_{f} .
$$

Then using Theorem 5.1 taking the Fréchet derivative of (31) and bringing all terms with $\dot{r}$ to the left hand side yields

$$
\dot{r}-D \Psi_{s_{f}}^{(2)}[\dot{r}]=\delta u_{f} \Delta[r]+D \Psi_{s}\left(s_{f}\right)^{(1)}[r] .
$$

Finally, by Lemma 6.1 .

$$
\begin{aligned}
\dot{r} & =\left(\mathrm{id}-D \Psi_{s_{f}}^{(2)}\right)^{-1}\left[\delta u_{f} \Delta[r]\right]+\left(\mathrm{id}-D \Psi_{s_{f}}^{(2)}\right)^{-1}\left[D \Psi_{s}\left(s_{f}\right)^{(1)}[r]\right] \\
& =: \mathcal{G}_{1}[r]+\mathcal{G}_{2}[r] .
\end{aligned}
$$

To compute the spectrum of $\mathcal{G}$ we proceed in several steps. First, we compute the resolvent of $\mathcal{G}_{1}$, in order to show the operator is self-adjoint and the real part of the spectrum is negative. Second, we use a Neumann series argument to ensure the spectrum of $\mathcal{G}_{1}$ does not shift too much when perturbed by $\mathcal{G}_{2}$. In this step, we see that for large enough $\delta$ the real part of the spectrum will remain negative.

Let $r \in H^{0}$ and $\lambda \in \mathbb{C}$ and consider

$$
\mathcal{G}_{1}[r]=\lambda r .
$$

Using the Fourier transform and Lemma 6.1 we solve for $\lambda$, which yields the eigenvalues of the operator defined as follows:

$$
\lambda_{k}:=-\delta u_{f}(2 \pi k)^{2}\left[\frac{2 \pi|k|\left(2+e^{-2 \pi|k| s_{f}}\right)}{1+4 \pi|k|+(2 \pi|k|-1) e^{-2 \pi|k| s_{f}}}\right], \quad k \in \mathbb{Z}^{*} .
$$

Since $s_{f}$ is positive and $u_{f}$ is nonnegative, (34) is strictly negative for all $k \in \mathbb{Z}^{*}$, and is bounded above by evaluating (34) at $k=1$. Thus,

$$
\lambda_{k} \leqslant-\delta u_{f}(2 \pi)^{2}\left[\frac{2 \pi\left(2+e^{-2 \pi s_{f}}\right)}{1+4 \pi+(2 \pi-1) e^{-2 \pi s_{f}}}\right]<0 \quad \forall k \in \mathbb{Z}^{*} .
$$

Let $f \in L^{0}$ and $\lambda \neq \lambda_{k}$ for $k \in \mathbb{Z}^{*}$. Then the resolvent of $\mathcal{G}_{1}$ is given by

$$
R\left(\lambda, \mathcal{G}_{1}\right): L^{0} \rightarrow H^{0} \subset L^{0}, \quad f \mapsto \mathcal{F}^{-1} \frac{1}{\lambda_{k}-\lambda} \mathcal{F}[f] .
$$


Since $H^{0}$ is dense in $L^{0}$, and $\mathcal{G}_{1}$ is a closed operator from $H^{0}$ into $L^{0}$, the adjoint, $\mathcal{G}_{1}^{*}$, exists and its resolvent satisfies

$$
R\left(\lambda, \mathcal{G}_{1}^{*}\right)=R\left(\bar{\lambda}, \mathcal{G}_{1}\right)^{*} \quad \forall \bar{\lambda} \in \rho\left(\mathcal{G}_{1}\right) .
$$

Taking advantage of the fact that we know $R\left(\lambda, \mathcal{G}_{1}\right)$ explicitly and the eigenvalues of $\mathcal{G}_{1}$ are all real, one observes that $\mathcal{G}_{1}$ is self-adjoint and its eigenvalues, $\lambda_{k}$, completely make up its spectrum. Next we show there exists a positive constant, $c$, such that the resolvent of $\mathcal{G}_{1}+\mathcal{G}_{2}$ contains a half-plane of $\mathbb{C}$ of the form $\{\lambda \in \mathbb{C}: \operatorname{Re}(\lambda) \geqslant-c\}$. Observe

$$
R\left(\lambda, \mathcal{G}_{1}+\mathcal{G}_{2}\right)=R\left(\lambda, \mathcal{G}_{1}\right)\left(\mathrm{id}-R\left(\lambda, \mathcal{G}_{1}\right) \mathcal{G}_{2}\right)^{-1}
$$

Thus $R\left(\lambda, \mathcal{G}_{1}+\mathcal{G}_{2}\right)$ exists as a bounded operator if we can show the inverse in (35) exists and is bounded. Fix $\delta$ positive and $\lambda$ such that $\operatorname{Re}(\lambda) \geqslant \lambda_{1} / 2$ so that

$$
\left\|R\left(\lambda, \mathcal{G}_{1}\right) \mathcal{G}_{2}\right\| \leqslant\left\|\frac{\lambda_{k}}{\lambda_{k}-\lambda} \frac{e^{-4 \pi|k| s_{f}}+(2 \pi|k|-1) e^{-2 \pi|k| s_{f}}-4 \pi|k|}{-\delta(2 \pi k)^{2}\left(2+e^{-2 \pi|k| s_{f}}\right)}\right\|_{l_{\infty}},
$$

yielding

$$
\left\|R\left(\lambda, \mathcal{G}_{1}\right) \mathcal{G}_{2}\right\| \leqslant \frac{2}{\delta} M
$$

where

$$
\begin{aligned}
M & =\left\|\frac{e^{-4 \pi|k| s_{f}}}{(2 \pi k)^{2}\left(2+e^{-4 \pi|k| s_{f}}\right)}\right\|_{l_{\infty}}+\left\|\frac{(2 \pi|k|-1) e^{-2 \pi|k| s_{f}}}{(2 \pi k)^{2}\left(2+e^{-4 \pi|k| s_{f}}\right)}\right\|_{l_{\infty}}+\left\|\frac{1}{(\pi k)\left(2+e^{-4 \pi|k| s_{f}}\right)}\right\|_{l_{\infty}} \\
& \leqslant \frac{5}{12 \pi^{2}}
\end{aligned}
$$

and

$$
\|\sigma(k)\|_{l_{\infty}}:=\sup _{k \in \mathbb{Z}^{*}}|\sigma(k)|
$$

From (36), if we choose $\delta>\delta_{0}:=5 /\left(6 \pi^{2}\right)$ we can construct the inverse operator in (35) via Neumann series resulting in $R\left(\lambda, \mathcal{G}_{1}+\mathcal{G}_{2}\right)$ as a bounded operator for all $\lambda$ such that $\operatorname{Re}(\lambda) \geqslant \lambda_{1} / 2$. In other words, we have shown

$$
\sup \{\operatorname{Re}(\lambda): \lambda \in \sigma(\mathcal{G})\} \leqslant-\left|\lambda_{1}\right| / 2<0
$$

To see $\mathcal{G}: H^{0} \subset L^{0} \rightarrow L^{0}$ is sectorial it is enough to check the boundedness of $\lambda R(\lambda, \mathcal{G})$ for all $\lambda$ such that $\operatorname{Re}(\lambda) \geqslant 0$ :

$$
\|\lambda R(\lambda, \mathcal{G})\| \leqslant\left\|\lambda R\left(\lambda, \mathcal{G}_{1}\right)\right\|\left\|\left(\mathrm{id}-R\left(\lambda, \mathcal{G}_{1}\right) \mathcal{G}_{2}\right)^{-1}\right\| \leqslant C, \quad C \in \mathbb{R}^{+},
$$

where the constant $C$ comes from the bound 36 and the definition of $R\left(\lambda, \mathcal{G}_{1}\right)$.

REMARK 1 Our choice to fix $\operatorname{Re}(\lambda) \geqslant \lambda_{1} / 2$ is clearly somewhat arbitrary and affects the size of $\delta_{0}$. Had we fixed $\lambda \geqslant c \lambda_{1}>0$ for $c \in(0,1 / 2)$ then $\delta_{0}$ would be smaller, entailing a more gentle exponential decay. In view of the complexity of the linearization, it is not clear how to avoid a perturbation argument and obtain stability for any $\delta>0$ as one naturally expects. 


\subsection{Proof of Theorem 3.1}

From Theorem 3.2 (local existence) we know that there exists a $t^{*}>0$ and a constant $C$ such that

$$
\left\|s(t, g)-s\left(t, g_{f}\right)\right\|_{C^{\alpha}\left(\left[0, t^{*}\right] ; H_{p}^{2}\right)} \leqslant C\left\|g-g_{f}\right\|_{H_{p}^{2}}
$$

so we define $r^{*}=s\left(t^{*}, g\right)-s\left(t^{*}, g_{f}\right)=s\left(t^{*}, g\right)-s_{f}$. Next, consider the system

$$
\begin{aligned}
\dot{r} & =\mathcal{G} r+\left(F\left[r+s_{f}\right]-F\left[s_{f}\right]-\mathcal{G} r\right), \\
r\left(t^{*}\right) & =r^{*},
\end{aligned}
$$

where $\mathcal{G}$ is defined in Lemma 6.2 and $r=s-s_{f}$. To see that (37) is equivalent to (31), recall $F\left[s_{f}\right]=u_{f}=\dot{s_{f}}$ and observe

$$
\begin{aligned}
\dot{s}-\dot{s_{f}} & =F[s]-\dot{s_{f}}=F[s]-F\left[s_{f}\right]-\mathcal{G}\left[s-s_{f}\right]-\dot{s_{f}}+F\left[s_{f}\right]+\mathcal{G}\left[s-s_{f}\right] \\
& =\mathcal{G}\left[s-s_{f}\right]+\left(F[s]-F\left[s_{f}\right]-\mathcal{G}\left[s-s_{f}\right]\right) .
\end{aligned}
$$

By Lemma 6.2 all assumptions of Theorem 9.1.2 in [12] are satisfied, and there exist constants $\omega, \tilde{\epsilon}, K$ such that if $\left\|r^{*}\right\|_{H^{2}} \leqslant \tilde{\epsilon}$, then the solution to 37)-38) exists globally and satisfies

$$
\|r(t)\|_{H_{p}^{2}} \leqslant K e^{-t \omega}\left\|r^{*}\right\|_{H_{p}^{2}} \quad \forall t \geqslant t^{*}
$$

where $\omega=\left|\lambda_{1}\right| / 2$. To complete the proof define $\epsilon=\tilde{\epsilon} / C$.

\section{Acknowledgments}

The first author was partially supported by the Goucher College Junior Faculty Development grant. Both authors wish to express their thanks to the anonymous referees for their constructive criticism.

\section{REFERENCES}

1. Alfrey, T., Gurnee, E. F., \& Lloyd, W. G. Diffusion in glassy polymers. J. Polymer Sci. 12 (1966), 249-261.

2. Astarita, G., \& Sarti, G. C. A class of mathematical models for sorption of swelling solvents in glassy polymers, Polymer Engrg. Sci. 18 (1978), 388-395.

3. Cohen, D. S., \& ERneux, T. Free boundary probelms in controlled release pharmaceuticals. I. Diffusion in glassy polymers. SIAM J. Appl. Math. 48 (1988), 1451-1465. Zbl 0704.35141 MR 0968840

4. Fasano, A., Meyer, G. H., \& Primicerio, M. On a problem in the polymer industry: theoretical and numerical investigation on swelling. SIAM J. Math. Anal. 17 (1986), 945-960. Zbl 0595.65134 MR 0846399

5. GuidotTi, P. Diffusion in glassy polymers: sorption of a finite amount of swelling solvent. Nonlinear Differential Equations Appl. 6 (1999), 297-317. Zbl 0947.35179|| MR 1710576

6. Guidoti, P. 2-D free boundary value problem with onset of a phase and singular elliptic boundary value problem. J. Evol. Equations 2 (2002), 395-424. Zbl 1040.35146 MR 1941034

7. Guidotti, P. A new first kind boundary integral formulation for the Dirichlet-to-Neumann map in 2D. J. Comput. Phys. 190 (2003), 325-345. Zbl 1030.65122 MR 2046767

8. Guidotti, P. A class of free boundary problems with onset of a new phase. SIAM J. Math. Anal. 38 (2007), 1981-2004. Zbl 1149.35084 MR 2299438 
9. Guidotti, P. Optimal regularity for a class of singular abstract parabolic equations. J. Differential Equations 232 (2007), 468-486. Zbl 1107.35073 MR 2286388

10. Guidotti, P., \& Palesko, J. Transient instability in Case II diffusion. J. Polymer Sci. B Polymer Phys. 36 (1998), 2941-2947.

11. Kumano-Go, H., \& Nagase, M. Pseudo-differential operators with non-regular symbols. Funkcial. Ekvac. 21 (1978), 151-192. Zbl 0395.35089 MR 0518297

12. Lunard, A. Analytic Semigroups and Optimal Regularity in Parabolic Problems. Birkhäuser (1995). Zbl 0816.35001 MR 1329547

13. Webster, M., \& Guidotti, P. Boundary dynamics of a two-dimensional diffusive free boundary problem. Discrete Contin. Dynam. Systems 26 (2010), 713-736. Zbl 1186.35249 MR 2556505 